\title{
Bayesian uncertainty analysis in distributed hydrologic modeling: A case study in the Thur River basin (Switzerland)
}

\author{
Jing Yang, ${ }^{1}$ Peter Reichert, ${ }^{1}$ and Karim C. Abbaspour ${ }^{1}$ \\ Received 3 September 2006; revised 1 May 2007; accepted 1 June 2007; published 2 October 2007. \\ [1] Calibration and uncertainty analysis in hydrologic modeling are affected by \\ measurement errors in input and response and errors in model structure. Recently, \\ extending similar approaches in discrete time, a continuous time autoregressive error \\ model was proposed for statistical inference and uncertainty analysis in hydrologic \\ modeling. The major advantages over discrete time formulation are the use of a \\ continuous time error model for describing continuous processes, the possibility of \\ accounting for seasonal variations of parameters in the error model, the easier treatment of \\ missing data or omitted outliers, and the opportunity for continuous time predictions. The \\ model was developed for the Chaohe Basin in China and had some features specific \\ for this semiarid climatic region (in particular, the seasonal variation of parameters in the \\ error model in response to seasonal variation in precipitation). This paper tests and extends \\ this approach with an application to the Thur River basin in Switzerland, which is subject \\ to completely different climatic conditions. This application corroborates the general \\ applicability of the approach but also demonstrates the necessity of accounting for the \\ heavy tails in the distributions of residuals and innovations. This is done by replacing the \\ normal distribution of the innovations by a Student $t$ distribution, the degrees of freedom \\ of which are adapted to best represent the shape of the empirical distribution of the \\ innovations. We conclude that with this extension, the continuous time autoregressive \\ error model is applicable and flexible for hydrologic modeling under different climatic \\ conditions. The major remaining conceptual disadvantage is that this class of approaches \\ does not lead to a separate identification of model input and model structural errors. The \\ major practical disadvantage is the high computational demand characteristic for all \\ Markov chain Monte Carlo techniques.
}

Citation: Yang, J., P. Reichert, and K. C. Abbaspour (2007), Bayesian uncertainty analysis in distributed hydrologic modeling: A case study in the Thur River basin (Switzerland), Water Resour. Res., 43, W10401, doi:10.1029/2006WR005497.

\section{Introduction}

[2] Because of measurement errors in input and response and errors in model structure, predictions of hydrologic models are inevitably affected by uncertainty. Hydrologic models play an important role in supporting environmental decisions, e.g., by assessing water availability, exploring vulnerability to environmental change, or predicting the effect of management measures in the watershed. Therefore, to be able to support environmental decisions under consideration of prediction uncertainty, careful analysis and quantification of uncertainty are crucial in hydrologic modeling.

[3] A significant number of techniques have been developed to estimate parameters and assess prediction uncertainty in hydrologic modeling. These include: first-order approximation [Carrera and Neuman, 1986; Kool and Parker, 1988; Vrugt and Bouten, 2002], Bayesian inference based on importance sampling (IS) [e.g., Kuczera and Parent, 1998] or Markov chain Monte Carlo (MCMC)

\footnotetext{
${ }^{1}$ Swiss Federal Institute of Aquatic Science and Technology, Duebendorf, Switzerland.
}

Copyright 2007 by the American Geophysical Union. 0043-1397/07/2006WR005497 [e.g., Vrugt et al., 2003, 2004; Kuczera and Parent, 1998], generalized likelihood uncertainty estimation (GLUE) [Beven and Binley, 1992], sequential uncertainty fitting (SUFI-2) [Abbaspour et al., 2004, 2007], parameter solution (ParaSol) [van Griensven and Meixner, 2006], and sources of uncertainty global assessment using split samples (SUNGLASSESS) [van Griensven and Meixner, 2006]. With respect to model results and their uncertainty bands, many applications of these techniques give similar results. However, there are differences in the statistical foundations of these techniques. Some of these techniques, such as GLUE [e.g., Beven and Binley, 1992] or SUFI-2 [Abbaspour et al., 2004, 2007], apply a philosophy that is not based on a statistical foundation (see, e.g., Beven [2006] for an explanation). On the other hand, applications of techniques that are based on a statistical foundation often use statistical assumptions, such as independent errors, which are obviously violated [e.g., Vrugt et al., 2003]. The violation of the statistical assumptions, particularly of homoscedasticity and independence of errors, is clearly and visually demonstrated by Vrugt et al. [2005]. Under such strong violations of the statistical assumptions, the derived parameter and prediction uncertainties are unreliable. As this is not a problem of the statistical inference 
procedure but of the formulation of the likelihood function, we think that the key of solving this problem is to improve the formulation of the likelihood function, rather than the development of new inference techniques with a poor conceptual foundation. The focus of such an improvement must be on the inclusion of input and model structure uncertainty in addition to parameter and output errors.

[4] Input and model structure uncertainty can be addressed by explicitly including these uncertainty sources into the formulation of the likelihood function, or by formulating an error model that jointly accounts for the effects of all uncertainty sources. There has been recently progressed in this research field. Kavetski et al. [2006] explicitly take into account input and output uncertainty in the formulation of the likelihood function. However, this approach does not consider the errors in model structure. Vrugt et al. [2005] present a simultaneous optimization and data assimilation (SODA) procedure to separate parameter uncertainty from input and model structural uncertainty. The main characteristic of SODA is to make the deterministic hydrologic model stochastic and combine parameter with state estimation. The difficulty of this approach is that it involves state estimation (which is equivalent to the estimation of many additional parameters) in addition to parameter estimation. This increases the computational burden and requires modifications to the existing simulation programs. A simpler approach to address input and model structural errors is by adding a "bias" or "model inadequacy" term to model output that provides a statistical description of the effect of model deficiencies on model output. This approach has recently gained attention in the literature [e.g., Kennedy and O'Hagan, 2001; Bayarri et al., 2007] in the context of interpolation (emulation) of the output of complex computer models. This approach is a more general formulation of the use of autoregressive error models to account for the effect of all error sources on the output of time series models, which has been applied frequently in hydrological modeling [e.g., Kuczera, 1983; Bates and Campbell, 2001]. Yang et al. [2007] further developed this discrete time overall additive autoregressive error model into a continuous time additive autoregressive error model and successfully applied it in the Chaohe Basin in China with the hydrological simulation program implemented in the soil and water assessment tool (SWAT) [Arnold et al., 1998]. This approach is an extension of the approach proposed by Duan et al. [1988] for unequally spaced data. In contrast to discrete time autoregressive error models, the continuous time autoregressive error model seems more satisfying because it can better describe the effects of input and model structural error that are of a continuous time nature, it makes it easier to describe seasonal dependence of error model properties, it eliminates the problems associated with missing data or omitted outliers, and it offers the opportunity for continuous time predictions [Yang et al., 2007]. This paper further tests this procedure by applying it to the Thur River basin in Switzerland. This is important to corroborate the universal applicability of the procedure under different climatic conditions and to gain experience with typical values of the parameters of the error model. In addition, we will extend the continuous time additive autoregressive error model by relaxing the assumption of normally distributed innovations to $t$-distributed innovations to account for the heavy tails of the distributions of innovations observed in the application to the Thur River basin.

[5] The remainder of this paper is organized as follows. In section 2, the continuous time autoregressive error model introduced by Yang et al. [2007] is described and extended. Section 3 will briefly describe the Thur River basin and the distributed hydrologic model implemented in the soil and water assessment tool [Arnold et al., 1998]. The results of the analysis are then discussed and compared to those for the Chaohe Basin [Yang et al., 2007] in section 4. Finally, a summary with conclusions is provided in section 5 .

\section{Bayesian Inference for a Continuous Time Autoregressive Error Model}

\subsection{Bayesian Inference}

[6] A deterministic hydrologic model can be written in the form of a function

$$
\mathbf{y}^{M}(\boldsymbol{\theta})=\left(y_{t_{0}}^{M}(\boldsymbol{\theta}), y_{t_{1}}^{M}(\boldsymbol{\theta}), \ldots, y_{t_{n}}^{M}(\boldsymbol{\theta})\right)
$$

where $y_{t_{i}}^{M}(\boldsymbol{\theta})$ represents the model output at time $t_{i}$ as a function of the model parameters $\boldsymbol{\theta}=\left(\theta_{1}, \ldots, \theta_{n}\right)$, and $M$ indexes the model.

[7] According to Bayes' theorem, the probability density of the posterior parameter distribution, $f_{\Theta \mid \mathbf{Y}}\left(\boldsymbol{\theta} \mid \mathbf{y}^{\text {obs }}\right)$, is derived from the prior density, $f_{\Theta_{\text {pri }}}(\boldsymbol{\theta})$, the likelihood function of the model, $f_{\mathbf{Y}^{M} \mid \Theta}\left(\mathbf{y}^{\text {obs }} \mid \boldsymbol{\theta}\right)$, and data, $\mathbf{y}^{\text {obs }}$, according to

$$
f_{\boldsymbol{\Theta} \mid \mathbf{Y}}\left(\boldsymbol{\theta} \mid \mathbf{y}^{\mathrm{obs}}\right)=\frac{f_{\mathbf{Y}^{M} \mid \boldsymbol{\Theta}}\left(\mathbf{y}^{\mathrm{obs}} \mid \boldsymbol{\theta}\right) \cdot f_{\boldsymbol{\Theta}_{\mathrm{pri}}}(\boldsymbol{\theta})}{\int f_{\mathbf{Y}^{M} \mid \boldsymbol{\Theta}}\left(\mathbf{y}^{\mathrm{obs}} \mid \boldsymbol{\theta}^{\prime}\right) f_{\boldsymbol{\Theta}_{\mathrm{pri}}}\left(\boldsymbol{\theta}^{\prime}\right) \mathrm{d} \boldsymbol{\theta}^{\prime}}
$$

Numerically, there are two generic Monte Carlo approaches to approximate the posterior parameter distribution (equation (2)), i.e., Markov Chain Monte Carlo (MCMC) and importance sampling (IS) [Gelman et al., 1995].

[8] In hydrology, the likelihood function is often constructed by assuming the residuals between the observations, $\mathbf{y}^{\text {obs }}$, and model results, $\mathbf{y}^{M}$, are identically, independently and normally distributed:

$$
f_{\mathbf{Y}^{M} \mid \boldsymbol{\Theta}}(\mathbf{y} \mid \boldsymbol{\theta})=\prod_{i=0}^{n}\left[\frac{1}{\sqrt{2 \pi}} \frac{1}{\sigma} \exp \left(-\frac{1}{2} \frac{\left[y_{t_{i}}-y_{t_{i}}^{M}(\boldsymbol{\theta})\right]^{2}}{\sigma^{2}}\right)\right]
$$

However, because of measurement errors in the model input and response and errors in model structure [Yang et al., 2007], this assumption is usually not satisfied and residuals are often heteroscedastic and autocorrelated. Therefore, in order to correctly apply Bayesian inference, the likelihood function must either address these errors explicitly or contain an autocorrelated component of residuals to describe their effect on model output.

\subsection{Additive Continuous Time Autoregressive Error Model}

[9] As an extension of the discrete time autoregressive error models introduced earlier [e.g., Kuczera, 1983; Bates and Campbell, 2001], an additive continuous time autore- 
gressive error model was introduced by Yang et al. [2007]. This model can account for heteroscedasticity and autocorrelation of residuals and it can easily handle missing data or omitted outliers. Briefly, the likelihood function is constructed as follows:

[10] For an autocorrelated random time series $E_{t_{1}}$ representing the effect of input, model structure and output errors we assume the probability density

$$
\begin{aligned}
f_{E_{t_{0}}}\left(\varepsilon_{t_{0}}\right)= & \frac{1}{\sqrt{2 \pi}} \frac{1}{\sigma} \exp \left(-\frac{1}{2} \frac{\varepsilon_{t_{0}}^{2}}{\sigma^{2}}\right) \\
f_{E_{t_{i}} \mid E_{t_{i-1}}}\left(\varepsilon_{t_{i}} \mid \varepsilon_{t_{i-1}}\right)= & \frac{1}{\sqrt{2 \pi}} \frac{1}{\sigma \sqrt{1-\exp \left(-2 \frac{t_{i}-t_{i-1}}{\tau}\right)}} \\
& \cdot \exp \left(-\frac{1}{2} \frac{\left(\varepsilon_{t_{i}}-\varepsilon_{t_{i-1}} \exp \left(-\frac{t_{i}-t_{i-1}}{\tau}\right)\right)^{2}}{\sigma^{2}\left(1-\exp \left(-2 \frac{t_{i}-t_{i-1}}{\tau}\right)\right)}\right)
\end{aligned}
$$

1992]. The same process was used by L. Tomassini et al. (A smoothing algorithm for estimating stochastic, continuous-time model parameters and an application to a simple climate model, submitted to Applied Statistics, 2007) to describe continuous, time-dependent model parameters. Note that the formulation of the continuous time error model (4) is similar to the approach suggested by Duan et al. [1988] for use with unequally spaced data. However, there is an essential difference between the two approaches: a decreasing temporal distance of measurement points in our error model leads not only to an increase of the correlation, but also to a decrease in the standard deviation of the error term. This guarantees continuity of the process realizations.

[12] Combining the deterministic hydrologic model (1) with the Box-Cox transformation [Box and Cox, 1964, 1982] and the error model (4), the likelihood function of the continuous time autoregressive error model can be written as

$$
\left.\begin{array}{rl}
f_{\mathbf{Y}^{M} \mid \boldsymbol{\Theta}}(\mathbf{y} \mid \boldsymbol{\theta})= & \frac{1}{\sqrt{2 \pi}} \frac{1}{\sigma} \exp \left(-\frac{1}{2} \frac{\left[g\left(y_{t_{0}}\right)-g\left(y_{t_{0}}^{M}(\boldsymbol{\theta})\right)\right]^{2}}{\sigma^{2}}\right) \cdot\left|\frac{d g}{d y}\right|_{y=y_{t_{0}}} \mid \\
& \cdot \prod_{i=1}^{n}\left[\frac{1}{\sqrt{2 \pi}} \frac{1 g\left(y_{t_{i}}\right)-g\left(y_{t_{i}}^{M}(\boldsymbol{\theta})\right)}{\sigma \sqrt{1-\exp \left(-2 \frac{t_{i}-t_{i-1}}{\tau}\right)}} \exp \left(-\frac{1}{2} \frac{\left.\left[g\left(y_{t_{i-1}}\right)-g\left(y_{t_{i-1}}^{M}(\boldsymbol{\theta})\right)\right] \exp \left(-\frac{t_{i}-t_{i-1}}{\tau}\right)\right]}{\sigma^{2}\left(1-\exp \left(-2 \frac{t_{i}-t_{i-1}}{\tau}\right)\right)}\right] \cdot\left|\frac{d g}{d y}\right|_{y=y_{t_{i}}}^{2} \mid\right.
\end{array}\right]
$$

where $\sigma$ is the asymptotic standard deviation of the errors and $\tau$ the characteristic correlation time. The assumption here is that the random disturbances, sometimes called innovations [Chatfield, 2003],

$$
I_{t_{i}}=E_{t_{i}}-E_{t_{i-1}} \exp \left(-\frac{t_{i}-t_{i-1}}{\tau}\right)
$$

rather than $E_{t_{i}}$, are independent and normally distributed. Keeping the asymptotic standard deviation of the errors $E_{t_{i}}$ at $\sigma$, the innovations must have standard deviations of

$$
\sigma_{I_{t_{i}}}=\sigma \sqrt{1-\exp \left(-2 \frac{t_{i}-t_{i-1}}{\tau}\right)}
$$

[11] They reach $\sigma$ if the time difference between two observations is large compared to the characteristic correlation time, $\tau$, and they are significantly smaller if succeeding observations are within that time or even closer. This error model is the analytical solution of an OrnsteinUhlenbeck stochastic process [e.g., Kloeden and Platen, where the function $g$ and $g^{-1}$ represent forward and backward Box-Cox transformations with parameters $\lambda_{1}$ and $\lambda_{2}$ :

$g(y)=\left\{\begin{array}{c}\frac{\left(y+\lambda_{2}\right)^{\lambda_{1}}-1}{\lambda_{1}} \lambda_{1}=0 \\ \ln \left(y+\lambda_{2}\right) \quad \lambda_{1}=0\end{array}\right.$
$g^{-1}(z)=\left\{\begin{array}{cc}\left(\lambda_{1} z+1\right)^{1 / \lambda_{1}}-\lambda_{2} & \lambda_{1}=0 \\ \exp (z)-\lambda_{2} & \lambda_{1}=0\end{array}, \frac{d g}{d y}=\left(y+\lambda_{2}\right)^{\lambda_{1}-1}\right.$

[13] In order to test the statistical assumptions of the likelihood function (7), a test should be made for the empirical distribution of the standardized observed innovations of the transformed observations $g\left(y_{\mathrm{t}_{\mathrm{i}}}^{\text {obs }}\right)$ and the transformed model results $g\left(y_{t_{i}}^{M}(\boldsymbol{\theta})\right)$ :

$$
\begin{aligned}
& i_{t_{i}}\left(\boldsymbol{\theta}, \mathbf{y}^{\mathrm{obs}}\right) \\
& =\frac{g\left(y_{\mathrm{t}_{\mathrm{i}}}^{\mathrm{obs}}\right)-g\left(y_{t_{i}}^{M}(\boldsymbol{\theta})\right)-\left(g\left(y_{\mathrm{t}_{\mathrm{i}-1}}^{\mathrm{obs}}\right)-g\left(y_{t_{i-1}}^{M}(\boldsymbol{\theta})\right)\right) \exp \left(-\frac{t_{i}-t_{i-1}}{\tau}\right)}{\sigma \sqrt{1-\exp \left(-2 \frac{t_{i}-t_{i-1}}{\tau}\right)}}
\end{aligned}
$$


[14] The suggested tests [e.g., Kuczera, 1983; Bates and Campbell, 2001; Yang et al., 2007] include plots of time series of innovations, of the autocorrelation function of innovations, of the cumulative periodogram, and of a normal quantile-quantile plot of the innovations.

\subsection{Error Model Extension}

[15] To be able to account for heavy tails of the innovations, we extend the assumption of normally distributed, independent innovations in equation (4) to independent $t$ distributions with the same standard deviations, i.e.,

$$
\begin{aligned}
& f_{E_{t_{0}}}\left(\varepsilon_{t_{0}}\right)=\frac{\Gamma\left(\frac{\nu+1}{2}\right)}{\Gamma\left(\frac{\nu}{2}\right)} \frac{1}{\sqrt{\pi(\nu-2)}} \cdot \frac{1}{\sigma}\left(1+\frac{\varepsilon_{t_{0}}^{2}}{(v-2) \sigma^{2}}\right)^{-\frac{v+1}{2}} \\
& f_{E_{t_{i}} \mid E_{t_{i-1}}}\left(\varepsilon_{t_{i}} \mid \varepsilon_{t_{i-1}}\right)=\frac{\Gamma\left(\frac{\nu+1}{2}\right)}{\Gamma\left(\frac{\nu}{2}\right)} \frac{1}{\sqrt{\pi(\nu-2)}} \\
& \cdot \frac{1}{\sigma \sqrt{1-\exp \left(-2 \frac{t_{i}-t_{i-1}}{\tau}\right)}} \\
& \left(1+\frac{\left(\varepsilon_{t_{i}}-\varepsilon_{t_{i-1}} \exp \left(-\frac{t_{i}-t_{i-1}}{\tau}\right)\right)^{2}}{(v-2) \sigma^{2}\left(1-\exp \left(-2 \frac{t_{i}-t_{i-1}}{\tau}\right)\right)}\right)^{-\frac{v+1}{2}}
\end{aligned}
$$$$
\text { for } \nu>2
$$

where $\Gamma$ denotes the gamma function and $\nu$ the degrees of freedom of the $t$ distribution (note that the degrees of freedom of the $t$ distribution must be larger than 2 in order to guarantee the existence of the standard deviation).

[16] Accordingly, the likelihood function is adapted to freedom leads us back to the previous assumption of normally distributed innovations.

\subsection{Uncertainty Analysis Procedure}

[18] Parameters to be estimated within the Bayesian framework with the autoregressive error model (equation (11)) include the parameters $\boldsymbol{\theta}$ of the hydrologic model, the parameters $\lambda_{1}$ and $\lambda_{2}$ of the Box-Cox transformation, the characteristic correlation time $\tau$, the standard deviation $\sigma$, and the degrees of freedom $\nu$ of the error model. Except $\nu$ which characterizes the shape of the $t$ distribution of the innovations, all of these parameters should be estimated jointly. This was done by applying a Markov chain Monte Carlo (MCMC) technique to approximate the posterior distribution of these parameters. In order to avoid long burn-in periods (or even lack of convergence to the distribution) of the Markov chain, the chain was started at a numerical approximation to the maximum of the posterior distribution calculated with the aid of the shuffled complex global optimization (SCE-UA) algorithm [Duan et al., 1992]. Markov chains were run until 20,000 model runs were reached with fulfillment of the convergence criterion by the Heidelberger and Welch [Cowles and Carlin, 1996; Best et al., 1995].

[19] The implementation of the modified likelihood function as well as the numerical realization of Bayesian inference was done in UNCSIM [Reichert, 2005].

\section{Study Area and SWAT Model}

\subsection{Description of the Study Area}

[20] The Thur River basin, with a drainage area of $1,700 \mathrm{~km}^{2}$, is situated in northeastern Switzerland near the border to Germany (Figure 1). Mean elevation of the watershed is about 774 meters above sea level and mean slope is

$$
\begin{aligned}
f_{\mathbf{Y}^{M} \mid \boldsymbol{\Theta}}(\mathbf{y} \mid \boldsymbol{\theta}, v)= & \frac{\Gamma\left(\frac{\nu+1}{2}\right)}{\Gamma\left(\frac{\nu}{2}\right)} \frac{1}{\sqrt{\pi(\nu-2)}} \frac{1}{\sigma}\left(1+\frac{\left[g\left(y_{t_{0}}\right)-g\left(y_{t_{0}}^{M}(\boldsymbol{\theta})\right)\right]^{2}}{(v-2) \sigma^{2}}\right)^{-\frac{v+1}{2}} \cdot\left|\frac{d g}{d y}\right|_{y=y_{t_{0}}} \mid \\
& \cdot \prod_{i=1}^{n}\left[\frac { \Gamma ( \frac { \nu + 1 } { 2 } ) } { \Gamma ( \frac { \nu } { 2 } ) } \frac { 1 } { \sqrt { \pi ( \nu - 2 ) } } \frac { 1 } { \sigma \sqrt { 1 - \operatorname { e x p } ( - 2 \frac { t _ { i } - t _ { i - 1 } } { \tau } ) } } \left(1+\frac{\left[g\left(y_{t_{i}}\right)-g\left(y_{t_{i}}^{M}(\boldsymbol{\theta})\right)-\left[g\left(y_{t_{i-1}}\right)-g\left(y_{t_{i-1}}^{M}(\boldsymbol{\theta})\right)\right] \exp \left(-\frac{t_{i}-t_{i-1}}{\tau}\right)\right]^{2}}{(v-2) \sigma^{2}\left(1-\exp \left(-2 \frac{t_{i}-t_{i-1}}{\tau}\right)\right)} \cdot\left|\frac{d g}{d y}\right|_{y=y_{t_{i}}}|| \mid\right.\right.
\end{aligned}
$$

The statistical tests to be used to assess the hypotheses of the error model are the plot of time series of innovations, autocorrelation functions of innovations, and $t$ distribution quantile-quantile plot of innovations.

[17] As the degrees of freedom $(\nu)$ approach infinity, the $t$ distribution will approximate the normal distribution. Therefore the additional flexibility of the error model provided by the degrees of freedom of the $t$ distribution extends our ability to approximate the observed distribution of innovations, while keeping the normal distribution as a limiting case. Lowering the degrees of freedom $(\nu)$ of the $t$ distribution leads to heavier tails, as it is often observed in hydrologic modeling. Increasing the number of degrees of around $7.5^{\circ}$. The climate of the watershed is the prealpine/ alpine climate, which is characterized by moderate winters in hilly dissected terrain area, cold winters in mountainous areas and summer seasons with relatively large annual temperature variations. Mean monthly temperature ranges from about $10^{\circ} \mathrm{C}$ to $25^{\circ} \mathrm{C}$ in the summer and from $-15^{\circ} \mathrm{C}$ to $7^{\circ} \mathrm{C}$ during the winter. The average precipitation is $1,460 \mathrm{~mm}$ $\mathrm{yr}^{-1}$ with high precipitation (about 2,200-2,500 $\mathrm{mm} \mathrm{yr}^{-1}$ ) in the mountain area and about $1,000 \mathrm{~mm} \mathrm{yr}^{-1}$ in the lower (submountain) part of the watershed, and most of precipita- 


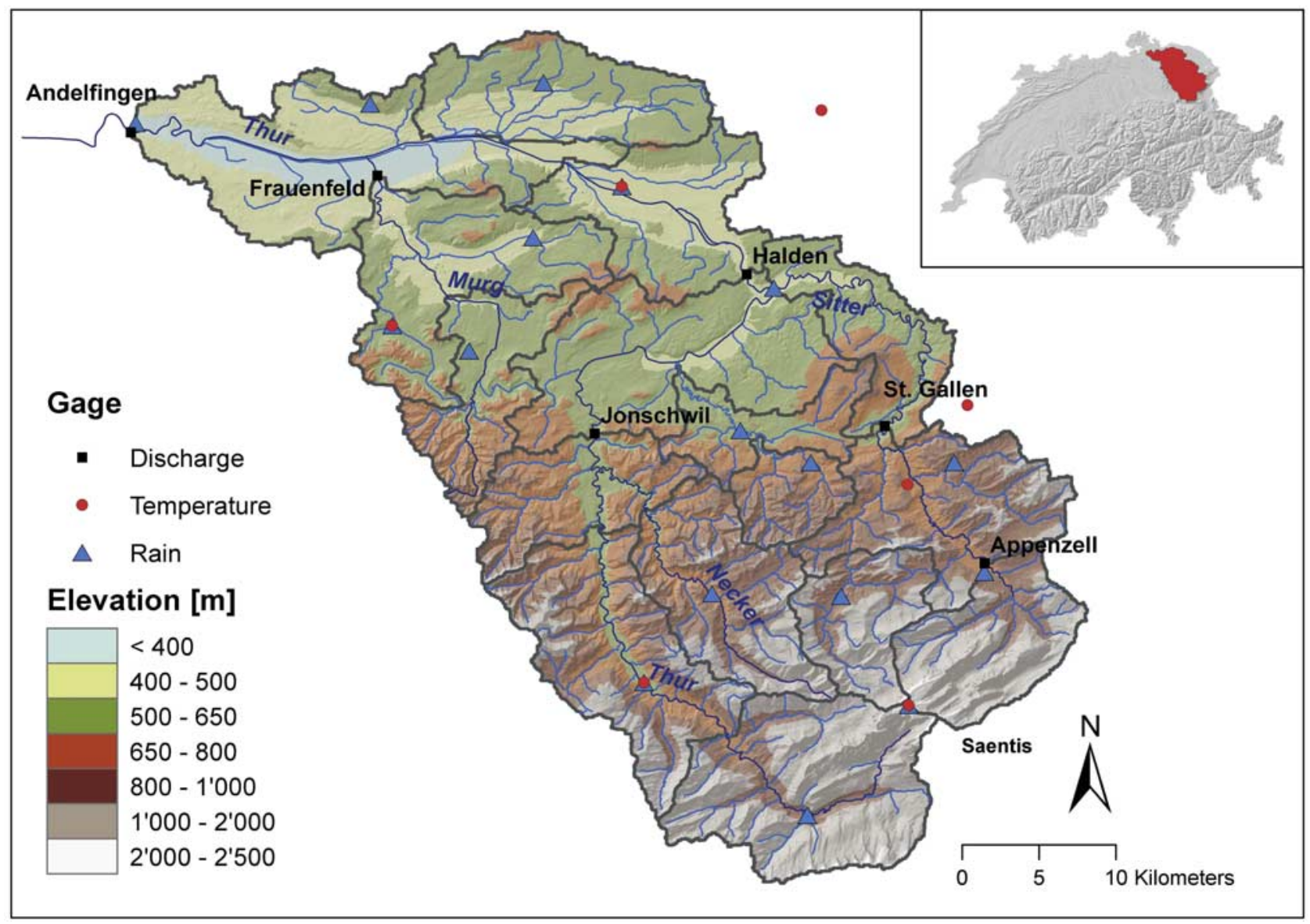

Figure 1. Thur River basin with SWAT-delineated subbasins, DEM map, river network, and meteorological stations. Reproduced with the permission of Swisstopo (BA067983).

tion falls during the summer months. The mean actual evapotranspiration is about $565 \mathrm{~mm} \mathrm{yr}^{-1}$, and runoff $895 \mathrm{~mm} \mathrm{yr}^{-1}$. The climate data used in this study are from seventeen precipitation, eight air temperature, five solar radiation, five relative humidity, and five wind speed gages (see Figure 1) over 20 years (1980-2000), which were obtained from the Swiss Federal Office of Meteorology and Climatology (http://www.meteoschweiz.ch/web/en/weather. html). The daily discharge is available at the basin outlet (Andelfingen station) from 1991 to 2000 from the Swiss Federal River Survey Program (NADUF; http://www.naduf.ch).

[21] The dominant land use (around 60\%) in this area is agriculture, most of which are meadows for feeding cows, alpine pastures, and arable lands. Close to $30 \%$ of the total area is covered by forests, about $3 \%$ are orchards. The rest of the area is barren land, surface waters, and urban areas. Hogs and cattle are the main livestock raised in the study area.

[22] Most of the Thur River basin is underlain by conglomerates, marl incrustations and sandstone with medium to low storage capacity and rather high permeability. Groundwater is mainly found in areas with till deposits [Gurtz et al., 1999].

\subsection{Description of SWAT and iSWAT}

[23] Soil and water assessment tool (SWAT) [Arnold et al., 1998; http://www.brc.tamus.edu/swat] implements a semidistributed and semiphysically based watershed model.
SWAT describes the climatic and topographic heterogeneity through subbasins based on a digital elevation map and climatic stations, while it describes the heterogeneities in land use, soil, and management practice through HRUs (hydrologic response units) which consist of unique combinations of land use, soil type, and management practice within the subbasin.

[24] At the HRU level, SWAT accounts for rainfall, interception, evapotranspiration, percolation, sediment yield, nutrient cycles, crop growth and management practice. Then, runoff, sediment yield and nutrient loads are aggregated to the subbasin level by taking the weighted average based on the areas of the HRUs. Water flow, sediment yield, and nutrient loading obtained at the subbasin level are then routed through the river system under consideration of in-stream transformation, deposition and remobilization processes. Channel routing is simulated using either the variable storage technique [Williams, 1969] or the Muskingum method [Cunge, 1969; Chow et al., 1988]. More detailed descriptions of the model can be found in the work by Arnold et al. [1998] and in SWAT manuals (available at http://www.brc.tamus.edu/swat).

[25] iSWAT is an interface between SWAT and an arbitrary system analysis tool that supports a simple, file-based interface [Reichert, 2006]. iSWAT was developed to facilitate the application of systems analysis techniques to hydrologic modeling on the basis of using SWAT [Yang et 
Table 1. Selected Parameters for Inference and Their Initial Values and Prior Distributions

\begin{tabular}{|c|c|c|c|}
\hline Aggregate Parameter ${ }^{\mathrm{a}}$ & $\begin{array}{l}\text { Name and Meaning of } \\
\text { Underlying SWAT Parameter }\end{array}$ & $\begin{array}{l}\text { Initial Parameter Range of } \\
\text { Underlying SWAT } \\
\text { Parameter }\end{array}$ & $\begin{array}{l}\text { Prior Distribution of } \\
\text { Aggregate Parameter }\end{array}$ \\
\hline $\mathrm{v}$ TIMP.bsn & Snowpack temperature lag factor & 0.307 & $\mathrm{U}[0.01,1]$ \\
\hline $\mathrm{v}$ SFTMP.bsn & Snowfall temperature & -1 & $\mathrm{U}[-5,5]$ \\
\hline $\mathrm{v}$ SMTMP.bsn & Snowmelt base temperature & 2.585 & $\mathrm{U}[-5,5]$ \\
\hline v_SMFMX.bsn & Melt factor for snow on $21 \mathrm{Jun}$ & 4.473 & $\mathrm{U}[0,10]$ \\
\hline $\mathrm{v}$ SMFMN.bsn & Melt factor for snow on $21 \mathrm{Dec}$ & 0.923 & $\mathrm{U}[0,10]$ \\
\hline v_MSK_CO1.bsn & $\begin{array}{l}\text { Muskingum coefficient to control impact of } \\
\text { the storage time constant for normal flow }\end{array}$ & 0 & $\mathrm{U}[0,10]$ \\
\hline v__MSK_CO2.bsn & $\begin{array}{l}\text { Muskingum coefficient to control impact of } \\
\text { the storage time constant for low flow }\end{array}$ & 0.2 & $\mathrm{U}[0,10]$ \\
\hline v_MSK_X.bsn & $\begin{array}{l}\text { A weighting factor that controls the relative } \\
\text { importance of inflow and outflow in } \\
\text { determining the storage in a reach } \\
\text { in Muskingum method }\end{array}$ & 0.1 & $\mathrm{U}[0,0.3]$ \\
\hline $\mathrm{v} \_$CH_K1.sub & $\begin{array}{l}\text { Effect hydraulic conductivity in tributary channel } \\
\text { alluvium, } \mathrm{mm} / \mathrm{hr}\end{array}$ & 0.5 & $\mathrm{U}[0,150]$ \\
\hline r CN2.mgt & CN2: curve number & $47-73$ & $\mathrm{U}[-0.35,0.35]$ \\
\hline $\mathrm{r}$ CH N2.rte & Manning roughness for main channel & $0.052 / 0.3$ & $\mathrm{U}[-0.5,0.5]$ \\
\hline v__ $\mathrm{CH}_{-} \mathrm{K} 2 . \mathrm{rte}$ & $\begin{array}{l}\text { Effective hydraulic conductivity in main channel } \\
\text { alluvium, } \mathrm{mm} / \mathrm{hr}\end{array}$ & 6.325 & $\mathrm{U}[0,150]$ \\
\hline v_ALPHA_BF.gw & Base flow alpha factor, $1 / \mathrm{d}$ & 0.0625 & $\mathrm{U}[0,1]$ \\
\hline V_GWQMN.gW & $\begin{array}{l}\text { Threshold depth of water in the shallow aquifer } \\
\text { required for return flow to occur, } \mathrm{mm} \mathrm{H}_{2} \mathrm{O}\end{array}$ & 0 & $\mathrm{U}[0,5000]$ \\
\hline v GW REVAP.gW & Groundwater "revap" coefficient & 0.02 & $\mathrm{U}[0.02,0.2]$ \\
\hline V_GW_DELAY.gW & Groundwater delay time, days & 43.338 & $\mathrm{U}[0,300]$ \\
\hline v CANMX.hru & Maximum canopy storage & 5.275 & $\mathrm{U}[0,10]$ \\
\hline v_ESCO.hru & Soil evaporation compensation factor & 0.154 & $\mathrm{U}[0,1]$ \\
\hline r_sOL_AWC.sol & Soil available water capacity, $\mathrm{mm} \mathrm{H}_{2} \mathrm{O} / \mathrm{mm}$ soil & $0-0.28$ & $\mathrm{U}[-0.5,0.5]$ \\
\hline $\mathrm{r}^{-} \mathrm{SOL}^{-} \mathrm{K} \cdot \mathrm{sol}$ & Soil hydraulic conductivity, $\mathrm{mm} / \mathrm{hr}$ & $0.01-279.71$ & $\mathrm{U}[-0.8,0.8]$ \\
\hline$\overline{\lambda_{1}} \mathrm{c}-{ }^{2}$ & Transformation factor in equations (7) or (11) & & $\begin{array}{l}\mathrm{U}[0,1] \\
\mathrm{U}[0,50]\end{array}$ \\
\hline$\lambda_{2}{ }^{\mathrm{c}}$ & Transformation factor in equations (7) or (11) & & $\mathrm{U}[0,50]$ \\
\hline$\sigma$ & Standard deviation in equations (7) or (11) & & Inv \\
\hline$\tau$ & $\begin{array}{l}\text { Characteristic correlation time of autoregressive } \\
\text { process, days }\end{array}$ & & Inv \\
\hline
\end{tabular}

aAggregate parameters are constructed on the basis of equation (12). For example, "v_ " in "v__TIMP.bsn" means "replace TIMP with a given value", and " $\mathrm{r}$ _" in "r_CN2.mgt" means "a relative change (of the default value) of CN2," and hence $\mathrm{r}$ _CN2.mgt is dimensionless.

${ }^{b} \mathrm{U}[\overline{\mathrm{x}, \mathrm{y}}]$ represents the uniform distribution over the interval $[\mathrm{x}, \mathrm{y}]$ for the given aggregate parameter; "Inv" denotes the probability distribution with probability density at the value $x$ proportional to $1 / x$.

${ }^{c}$ Here $\lambda_{1}$ and $\lambda_{2}$ are fixed to 0 and are excluded in the final MCMC as they are very close to 0 .

al., 2005]. In $i S W A T$, SWAT parameters can be aggregated on the basis of important influential factors, such as land use, soil texture, soil hydrologic group or subbasin as follows:

$$
\begin{aligned}
& \mathrm{x}_{\ldots}<\text { parname }>.<\text { ext }>\ldots<\text { hydrogrp }>\ldots<\text { soltext }>\text { _ } \\
& <\text { landuse }>\text { _ }<\text { subbsn }>
\end{aligned}
$$

where $\mathrm{x}$ represents the type of change to be applied to the parameter (v: value; a: absolute change; or $r$ : relative change), < parname $>$ is the SWAT parameter name; <ext $>$ represents the extension of the SWAT input file which contains the parameter, $<$ hydrogrp $>$ is the identifier for the hydrologic group, $<$ soltext $>$ is the soil texture, $<$ landuse $>$ is the land use, and $<$ subbsn $>$ is the subbasin number, or the crop index, or the fertilizer index. For example, $\mathrm{v}$ CN2.mgt $=69$, will cause a global replacement of $\overline{\mathrm{CN}} 2$ value in the management files by 69 , and r CN2.mgt 23,25 $\quad 23$, will cause a replacement of the CN2 value in the management files associated with subbasins 23 and 25 by a value equal to their current CN2 values multiplied by $(1+0.3)$, etc.

\subsection{Choice of Parameters and Priors}

[26] After setting up the project, a manual calibration and then an automatic calibration were done on some parameters of the Thur SWAT project. All the simulations in this paper are based on the calibrated project for all parameters not included in the analysis (i.e., not explicitly mentioned). To distinguish these simulations from the following new simulations, they are referred to as "previous simulations" in the following text.

[27] The choice of parameters is based on the Latinhypercube-one-factor-at-a-time (LH-OAT) method [van Griensven et al., 2006]. LH-OAT is a global screening sensitivity analysis technique and its characteristic is that it combines the Latin hypercube sampling [McKay et al., 1979] and one-factor-at-a-time (OAT) method by taking the Latin hypercube samples as initial points for the OAT method. On the basis of LH-OAT, 20 aggregate SWAT parameters related to river flow were selected for calibration (Table 1).

[28] Together with the parameters $\lambda_{1}, \lambda_{2}, \sigma$ and $\tau$ of the autoregressive error model in equations (7) or (11), there are 24 parameters. The prior distributions of all these parameters are assumed to be independent. For the 20 aggregate 

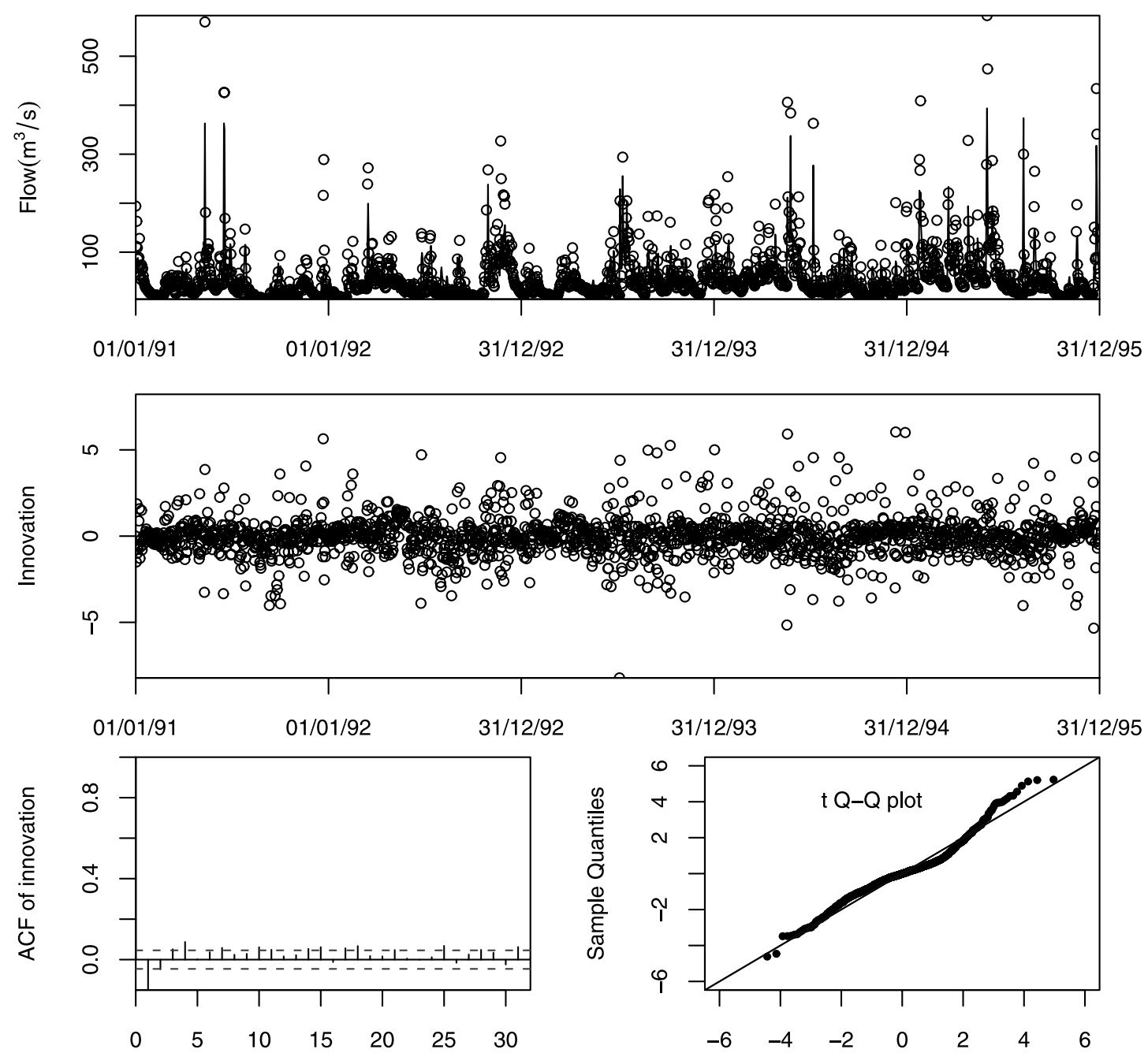

Lag

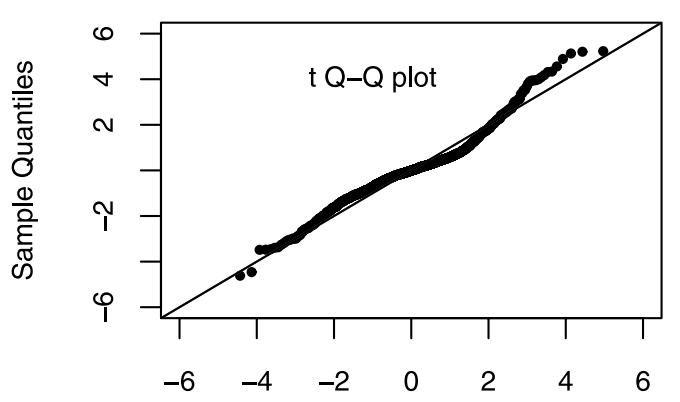

Theoretical t-distribution Quantiles

Figure 2. Statistics diagnostics for simulation 1 with likelihood function (11) with degrees of freedom 8. (top) Time series of the observed (circles) and simulated (line) flows, (middle) time series of the normalized innovations, and (bottom) autocorrelation function and $t$ distribution quantile-quantile plot of normalized innovations.

SWAT parameters, uniform priors with reasonable ranges were assumed (see the last column in Table 1) as "this may not always be easy to assess a priori" even for the soil hydraulic conductivity [Beven and Binley, 1992, p. 286]. In addition, transformation parameters $\lambda_{1}$ and $\lambda_{2}$ are assumed to be uniformly distributed. For parameters $\sigma$ and $\tau$, densities proportional to $1 / \sigma$ and $1 / \tau$ were chosen. Table 1 gives an overview of the parameters used for calibration and their prior distribution.

[29] Except for the analysis with likelihood function (11), 2 additional analyses with likelihood functions (3) and (7) were also carried out as a comparison to the analysis on the basis of the likelihood function (11). It is worth noting that the likelihood function (3) is widely used in hydrology (and many other fields) and the likelihood function (7) was used by Yang et al. [2007]. Hereafter, simulations based on analyses with likelihood functions (11), (3) and (7) are referred to as simulation 1 , simulation 2 and simulation 3 , respectively.
[30] Obviously the initial values of storage volumes (e.g., soil water content) will influence the river flow. As we cannot specify reasonable initial values for all storage volumes considered in the model, SWAT is operated for a "warm-up period" of 6 years (1985-1990) without comparison of model results with observed data. We found that such a "warm-up period" was sufficient to minimize the effects of the initial state of SWAT variables on river flow. Furthermore, in order to verify the calibrated model parameters, the model was calibrated and tested on the basis of the observed discharges at the basin outlet (Andelfingen station, Figure 1) using a split sample procedure. The data from the years 1991-1995 was used for calibration, and the data from 1996-2000 was used to test the model.

\section{Results and Discussion}

\subsection{Results for the Thur River Basin}

[31] To determine the optimum value of the degrees of freedom, $\nu$, of the $t$ distribution in the likelihood function 

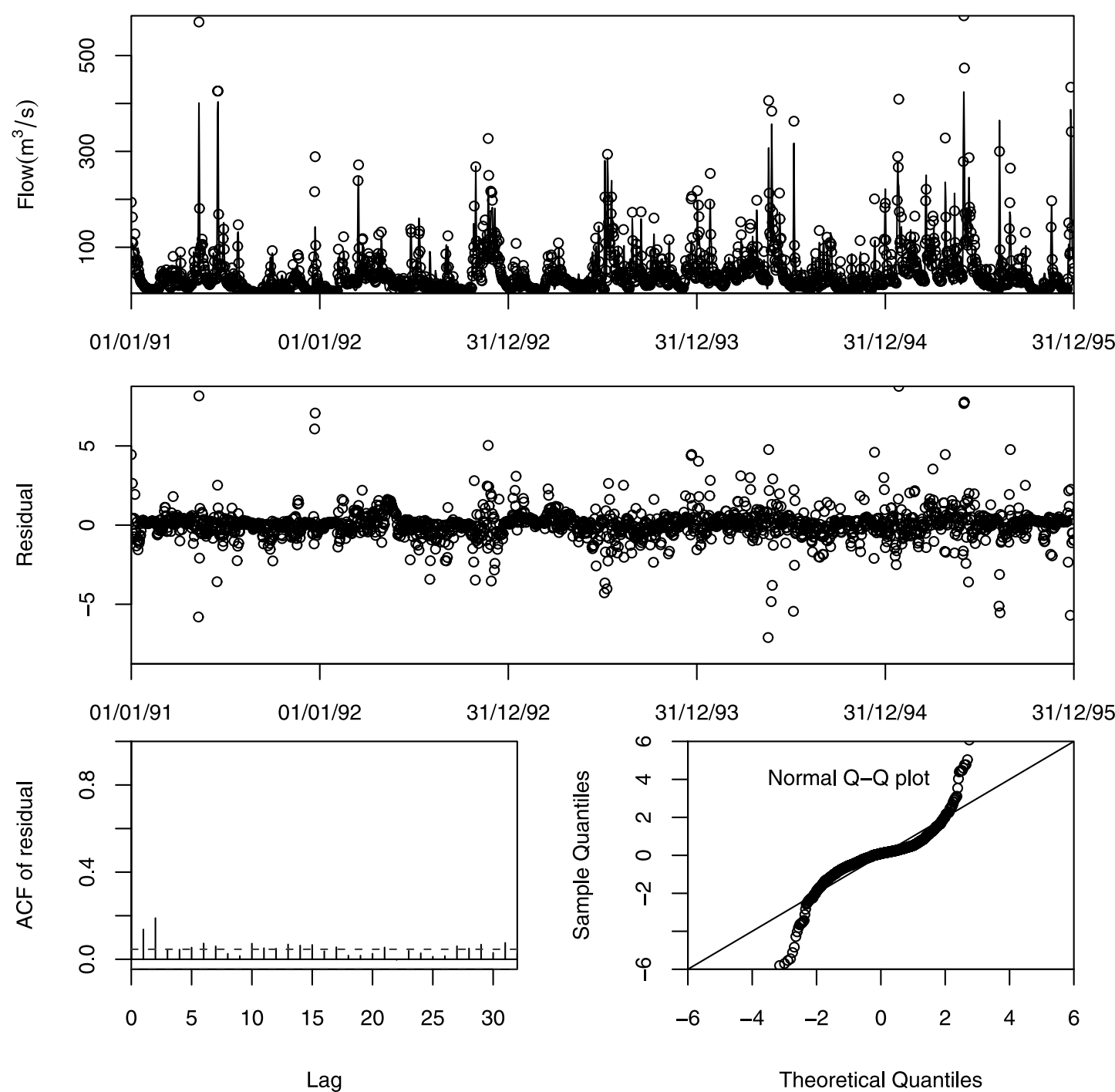

Figure 3. Statistics diagnostics for simulation 2 with likelihood function (3). (top) Time series of the observed and simulated flows, (middle) time series of the normalized residuals, and (bottom) autocorrelation function and normal quantile-quantile plot of normalized residuals.

given by equation (11), we compared regression diagnostics for analyses performed with different values of $\nu$. The comparisons were done for simulation results at the maximum of the posterior density obtained with the aid of the global optimization algorithm SCE-UA [Duan et al., 1992]. The comparisons showed that the simulation with $\nu=8$ led to the smallest deviations of the residuals from the theoretical assumptions made by the model. These results of regression diagnostics with $\nu=8$ are illustrated in Figure 2. The top plot in Figure 2 shows the time series of observed (circles) and simulated (line) flows. For this simulation, $\mathrm{R}^{2}$ equals 0.80 and the Nash-Sutcliffe coefficient equals 0.77 . The middle plot in Figure 2 shows the time series of the innovations. There seems to be no serious violation of the assumptions of independence and of distribution shape. This is further corroborated by the autocorrelation function (bottom left plot) and the $t$ distribution quantile-quantile plot (bottom right plot). The autocorrelations are very small except for the first-order coefficient. The quantile-quantile plot in the bottom right plot demonstrates that the empirical quantiles of the innovations are in good agreement with the theoretical $t$ distribution quantiles.

[32] For comparative purposes, Figures 3 and 4 show the corresponding results and diagnostics for analyses of simulations 2 and 3, respectively, and Table 2 lists the performances of 3 simulations at the maxima of posterior densities. The top plots in Figures 2, 3, and 4 give the impression that all three simulations led to similarly good agreement with data, although simulation 2 captured several peaks better than simulations 1 and 3 (e.g., flow at 1991-8-22). This led to the highest $\mathrm{R}^{2}$ and the Nash-Sutcliffe coefficient calculated with the simulated flow and observed flow (Table 2). This is because the Box-Cox transformation with $\lambda_{1}=0$ (the optimized $\lambda_{1}$ s in simulations 1 and 3 are very close to 0 ) puts less weight on the good approximation of high peaks to account for the lower measurement accuracy. However, the significant heteroscedasticity in the residuals of simulation 2 violates the statistical assumptions and makes its uncertainty estimates unreliable (middle plot of Figure 3). There are also slightly higher autocorrelation coefficients (bottom left 

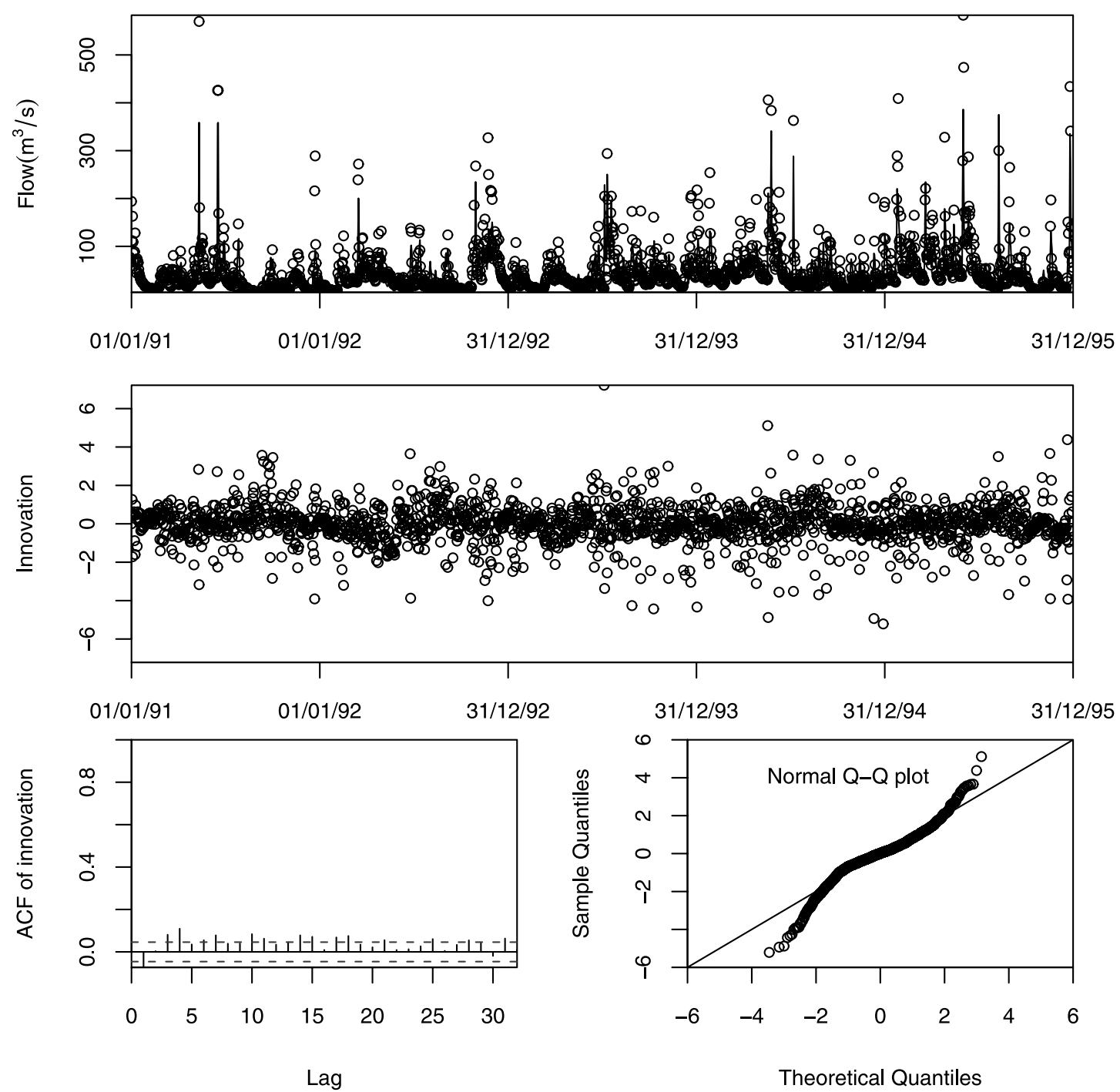

Figure 4. Statistics diagnostics for simulation 3 with likelihood function (7). (top) Time series of observed and simulated flows, (middle) time series of normalized innovations, and (bottom) autocorrelation function and normal quantile-quantile plot of innovations.

plot of Figure 3), and the assumption of normally distributed residuals is severely violated especially in the tails of the distribution (bottom right plot of Figure 3). Also for simulation 3, the distribution of the innovations is far from normal (especially in the tails) although better than that of simulation 2 (bottom right plot of Figure 4). In conclusion, simulation 1 is the only one that does not significantly violate its statistical assumptions. In Table 2, simulation 2 obtained the highest values $\mathrm{R}^{2}$ and Nash-Sutcliffe coefficient calculated with the simulated flow and observed flow. This demonstrates that unweighted least squares regression is an efficient technique to find a good fit solution. However, as mentioned above, this technique cannot be used to get reliable uncertainty estimates of model parameters and results.

[33] For simulation 1, a Markov chain was started from the approximation to the maximum of the posterior density to get an approximation to the posterior distribution. The preliminary Markov chain led to the conclusion that both $\lambda_{1}$ and $\lambda_{2}$ are very close to 0 . To decrease the complexity of the
MCMC process, we fixed $\lambda_{1}$ and $\lambda_{2}$ to 0 and excluded them from further MCMC processes. After a burn-in period of 40,000 model runs, 20,000 model runs were used to obtain the posterior parameter distribution and prediction uncertainty.

[34] Figure 5 shows the marginal distributions of the posterior parameter distribution. The increase in $\mathrm{CN} 2$ (positive value of $\mathrm{r}$ CN2.mgt) reflects higher surface runoff than in the previous simulation, while an increase in ESCO (value around 0.32 instead of 0.154 ) indicates smaller evapotranspiration than in the previous simulation. The changes in the temperature related parameters (TIMP, SFTMP, SMTMP, SMFMX and SMFMN) demonstrate that temperature factors have a significant influence on river flow. The marginal posteriors of some parameters (e.g., $\mathrm{v}$ TIMP.bsn and $\mathrm{r} \quad \mathrm{CH}$ N2.rte) are at the boundary of the prior interval. This can be an indication for very poor identifiability due to strong correlations in the posterior. The large increase in $\mathrm{CH} \mathrm{K} 2$ reflects a stronger interaction between channel and groundwater. The characteristic cor- 
Table 2. Performance of Three Simulations at the Maxima of the Posterior Distribution

\begin{tabular}{|c|c|c|c|}
\hline Test Data & Nash-Sutcliffe & $\mathrm{R}^{2}$ & Log Posterior Density \\
\hline \multicolumn{4}{|c|}{ Simulation 1 With Likelihood Function (11) } \\
\hline Calibration period & 0.77 & 0.80 & -6510 \\
\hline Validation period & 0.79 & 0.82 & -6586 \\
\hline \multicolumn{4}{|c|}{ Simulation 2 With Likelihood Function (3) } \\
\hline Calibration period & 0.85 & 0.85 & -8615 \\
\hline Validation period & 0.86 & 0.86 & -8597 \\
\hline \multicolumn{4}{|c|}{ Simulation 3 With Likelihood Function (7) } \\
\hline Calibration period & 0.77 & 0.80 & -6668 \\
\hline Validation period & 0.79 & 0.83 & -6742 \\
\hline
\end{tabular}
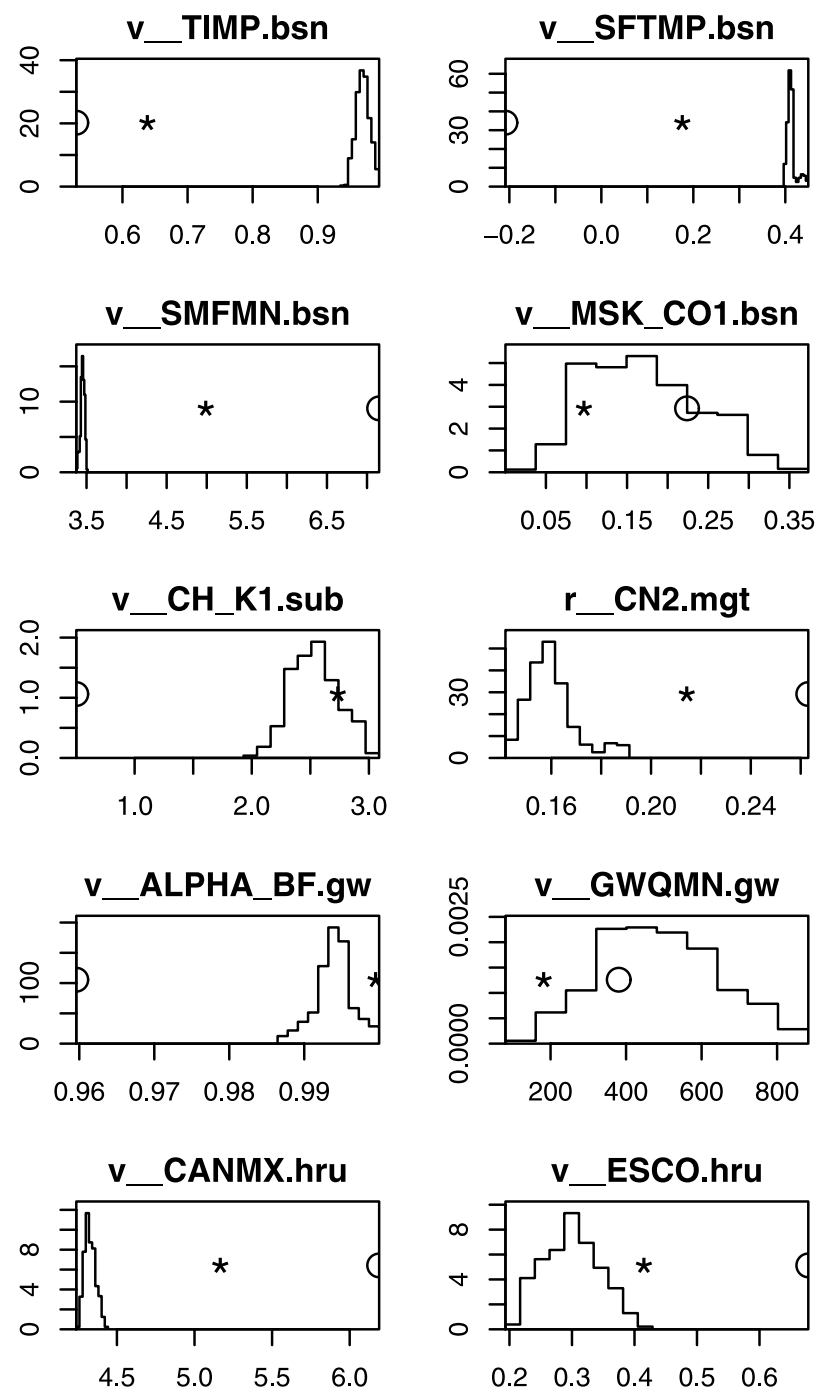

$\sigma$
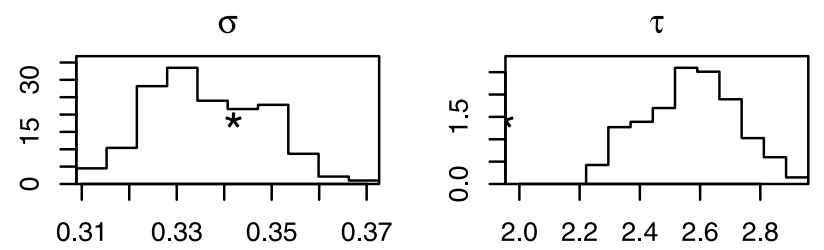

relation time is around 2 days. This indicates that there is no long-term correlation in the residuals. The parameter values corresponding to the maximum posterior density for simulations 2 and 3 are also plotted in Figure 5 as circles and asterisks, respectively. As we can see, because of different objective functions, optimized parameter values vary a lot.

[35] Figure 6 shows the $95 \%$ prediction uncertainty bands associated with parameter uncertainty (dark shaded area), and with parameter uncertainty and continuous time autoregressive error model uncertainty (light shaded area) for both calibration period and validation period. As can be seen, although the prediction uncertainty from parameter uncertainty (dark shaded area) is very narrow (it only covers $7.2 \%$ of the observations), the $95 \%$ uncertainty bands from parameter uncertainty and autoregressive error model
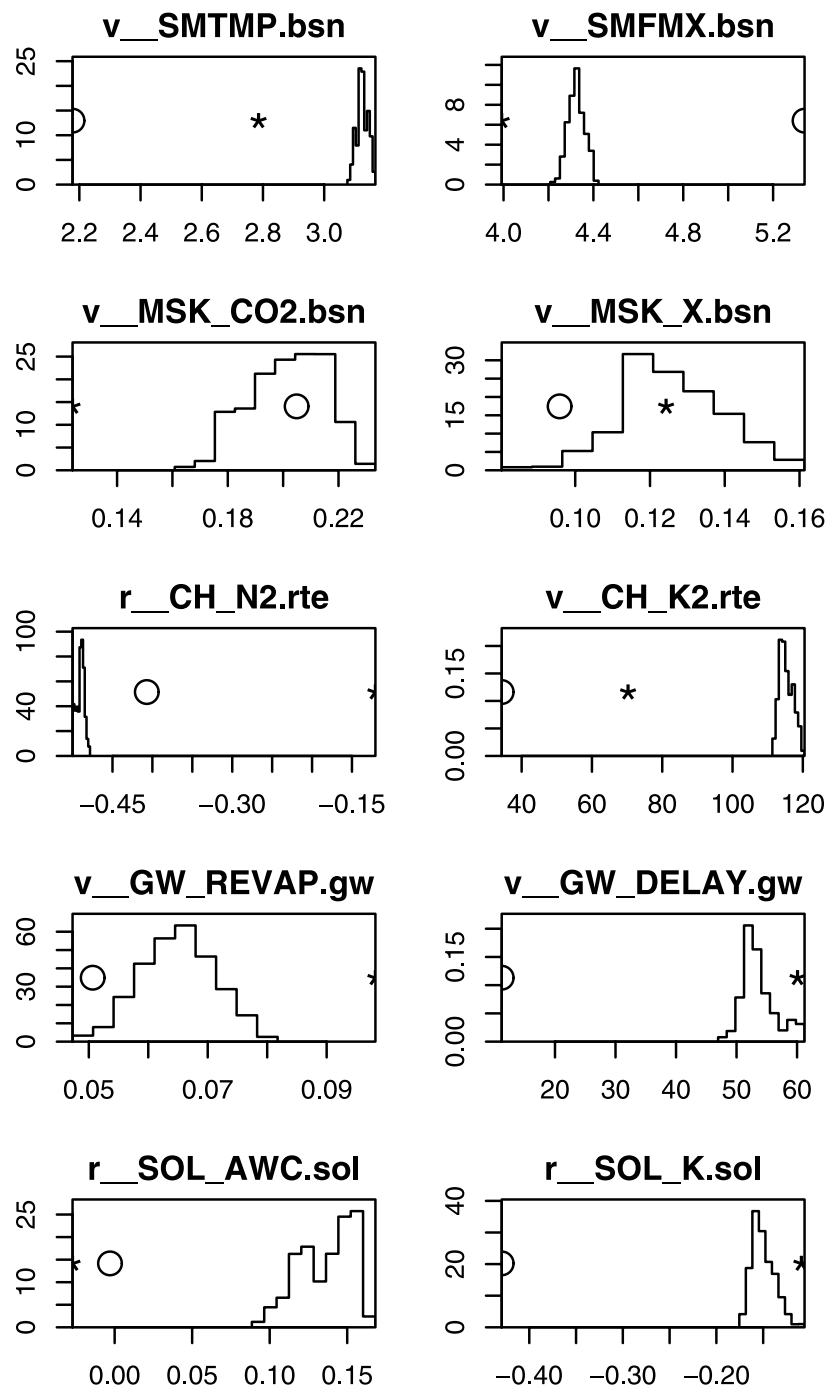

Figure 5. Histograms approximating the marginals of the posterior parameter distribution for simulation 1 and optimized parameters for simulation 2 (circles) and simulation 3 (asterisks). 

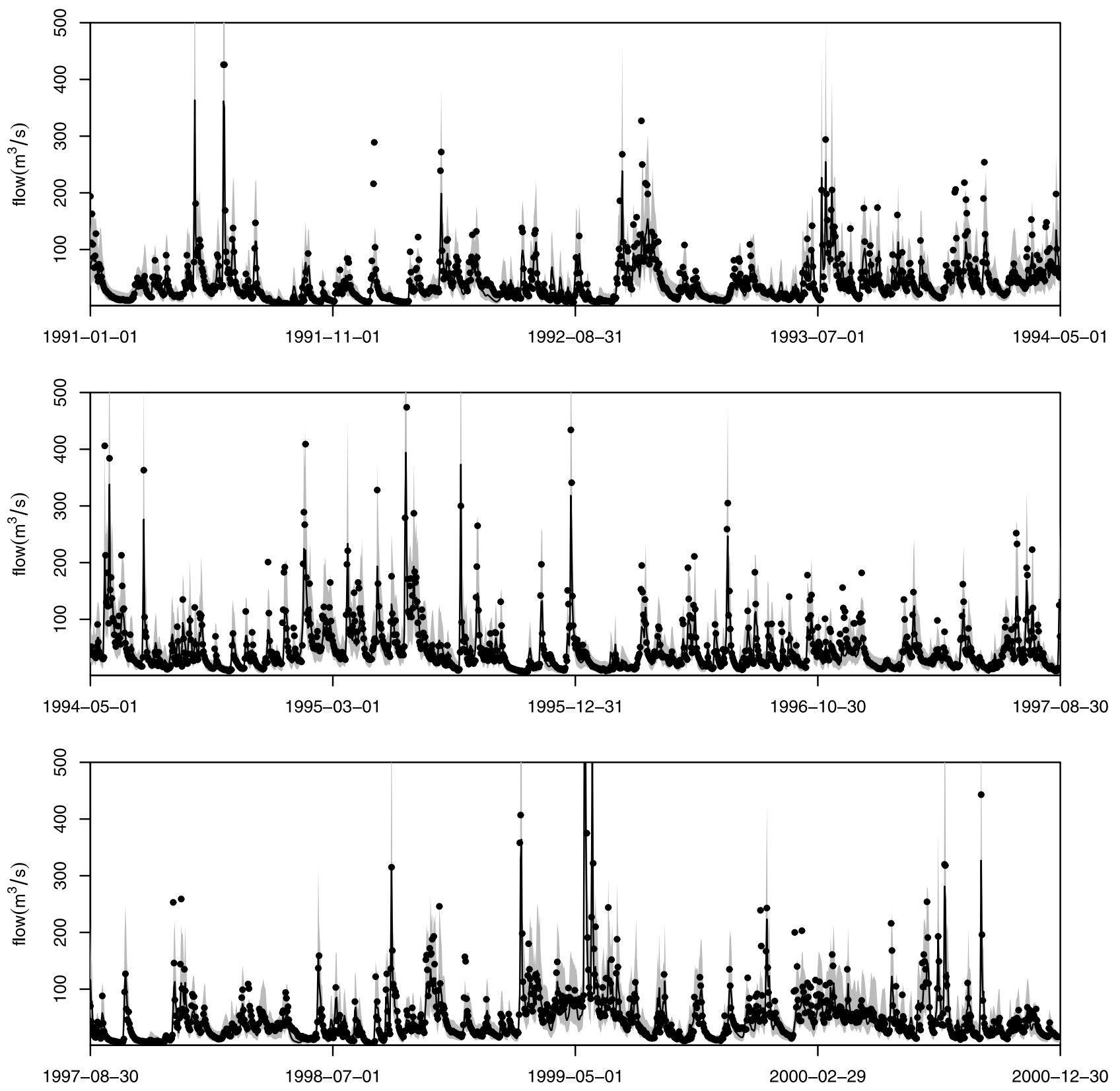

Figure 6. Plots of $95 \%$ prediction uncertainty bands associated with parameter uncertainty (dark shaded area) and with parameter uncertainty and continuous time autoregressive error model (light shaded area) for simulation 1 for both calibration period (1991-1995) and test period (1996-2000). The dots correspond to the observed flow series at the basin outlet, and the line stands for the simulated discharge at the maximum of the posterior distribution.

brackets most of the observations, which indicates that our proposed approach can mimic the prediction uncertainty (it covers $92.3 \%$ of the observations). The dots correspond to the observed discharge at the basin outlet and the line represents the simulated discharge at the maximum of the posterior distribution.

\subsection{Comparison With the Results of the Chaohe Basin}

[36] Compared to the application of the continuous time autoregressive error model in the Chaohe Basin in China by Yang et al. [2007], we can find some differences and similarities:
[37] 1. SWAT parameters: In the Chaohe Basin, river discharge is only sensitive to runoff generation (e.g., CN2, SOL_AWC and ESCO) during the wet season and the snow accumulation and melting processes are negligible. In the Thur River basin, flow is not only due to runoff generation (e.g., CN2, SOL_AWC and ESCO), but snow accumulation and melting processes are relevant (e.g., TIMP, SFTMP, SMTMP, SMFMX and SMFMN).

[38] 2. Standard deviation $(\sigma)$ and characteristic correlation time $(\tau)$ of the error model: In the Chaohe Basin, these 2 parameters have a strong seasonal dependence, i.e., high $\sigma$ and low $\tau$ during the wet season, and low $\sigma$ and very high $\tau$ during the dry season. In the Thur River basin $\tau$ is relatively 
small. This can be explained by the climate difference of these 2 basins. In the temperate continental and semiarid climate in the Chaohe Basin with over $80 \%$ rainfall in July and August, the flow during the dry weather season is strongly dependent on the water stored during the wet season. This leads to the very high value of the correlation time during the dry season. In the prealpine/alpine climate in the Thur River basin, river discharge is much more strongly dependent on rain events distributed throughout the year.

[39] 3. Prediction uncertainty: No matter how the continuous time autoregressive error model is applied, the characteristic of the prediction is the same: narrow prediction uncertainty band from parameter uncertainty and substantially wider prediction uncertainty band from the continuous time autoregressive error model. This difference between the 2 uncertainty bands indicates a high fraction of uncertainty due to input and model structure. The uncertainty due to parameters of the deterministic models may be underestimated by this procedure.

[40] 4. Convergence of MCMC: The Markov chain for the simulation of the Thur River basin converged slower than that of the Chaohe Basin. The reason might be that the number of parameters in the simulation of the Thur River basin is large and the shape of the posterior is more complicated than that of the Chaohe Basin (possibly multimodal with many local maxima).

\section{Summary and Conclusion}

[41] The continuous time autoregressive error model developed by Yang et al. [2007] for hydrologic modeling was tested for a watershed with completely different characteristics than the one of Yang et al. [2007]. This application required an extension of the distributional shape of the innovations from a normal distribution to a Student $t$ distribution to account for heavier tails of the innovations. The extended error model was successfully applied (empirical results are not in disagreement with distributional assumptions made by the model) to an implementation of the hydrologic model of the soil and water assessment tool (SWAT) [Arnold et al., 1998] for the Thur River basin in Switzerland. The results for the Thur River basin are compared to those for the Chaohe Basin in China described in the previous paper fs[Yang et al., 2007].

[42] These analyses led to the following conclusions:

[43] 1. Our case studies indicate that the extended continuous time autoregressive error model is generally applicable as an error model for hydrologic simulations under significantly different climatic conditions (case studies for semiarid climate in North China and prealpine/alpine climate in Switzerland). This was confirmed by statistical tests of the distributional assumptions of the model.

[44] 2. Two case studies indicate that the parameters of the hydrologic model as well as the parameters of the error model need careful site-specific priors and calibration. Particularly, the degrees of freedom of the $t$ distribution proved to be an effective parameter to adjust the distributional shape of the innovations (to account for heavy tails), and the standard deviation and characteristic correlation time of the error model required a seasonal variation for the semiarid climate in North China that was not required under the prealpine/alpine climate in Switzerland. The reason for this is that river discharge during very long dry weather periods is dependent on precipitation during the rainy season before, whereas the dominant influence on river discharge during wet periods is rain event over a much shorter preceding period.

[45] While our approach leads to a satisfactory mechanistic and statistical description of runoff, it does not separate input and model structural uncertainty. The resolution of this should continue to be a future effort in hydrological systems analysis.

[46] Acknowledgments. We thank the Swiss Federal Office of Meteorology and Climatology (http://www.meteoschweiz.ch/web/en/weather. html) and the Swiss Federal River Survey Program (NADUF; http:// www.naduf.ch) for the provision of climatological and river discharge data.

\section{References}

Abbaspour, K. C., C. A. Johnson, and M. T. van Genuchten (2004), Estimating uncertain flow and transport parameters using a sequential uncertainty fitting procedure, Vadose Zone J., 3(4), 1340-1352.

Abbaspour, K. C., J. Yang, I. Maximov, R. Siber, K. Bogner, J. Mieleitner, Z. Jobrist, and S. Raghavan (2007), Spatially-distributed modelling of hydrology and water quality in the pre-alpine/alpine Thur watershed using SWAT, J. Hydrol., 333, 413-430.

Arnold, J. G., R. Srinivasan, R. S. Muttiah, and J. R. Williams (1998), Large area hydrologic modeling and assessment-part 1: Model development, J. Am. Water Resour. Assoc., 34(1), 73-89.

Bates, B. C., and E. P. Campbell (2001), A Markov chain Monte Carlo scheme for parameter estimation and inference in conceptual rainfallrunoff modeling, Water Resour. Res., 37(4), 937-947.

Bayarri, M. J., J. O. Berger, R. Paulo, J. Sacks, J. A. Cafeo, J. Cavendish, C. H. Lin, and J. Tu (2007), A framework for validation of computer models, Technometrics, 49(2), 138-154.

Best, N. G., M. K. Cowles, and S. K. Vines (1995), Convergence diagnosis and output analysis software for Gibbs sampler output: Version 0.3, Med. Res. Counc. Biostat. Unit, Cambridge, U. K.

Beven, K. (2006), A manifesto for the equifinality thesis, J. Hydrol., 320, 18-36.

Beven, K., and A. Binley (1992), The future of distributed models-Model calibration and uncertainty prediction, Hydrol. Processes, 6(3), 279298.

Box, G. E. P., and D. R. Cox (1964), An analysis of transformations, J. R. Stat. Soc., Ser. B, 26, 211-252.

Box, G. E. P., and D. R. Cox (1982), An analysis of transformations revisited, rebutted, J. Am. Stat. Assoc., 77(377), 209-210.

Carrera, J., and S. P. Neuman (1986), Estimation of aquifer parameters under transient and steady-state conditions: 1. Maximum-likelihood method incorporating prior information, Water Resour. Res., 22(2), $199-210$.

Chatfield, C. (2003), The Analysis of Time Series: An Introduction, 6th ed., Chapman and Hall, New York.

Chow, V. T., D. R. Maidment, and L. W. Mays (1988), Applied Hydrology, McGraw-Hill, New York.

Cowles, M. K., and B. P. Carlin (1996), Markov chain Monte Carlo convergence diagnostics: A comparative review, J. Am. Stat. Assoc., 91(434), 883-904.

Cunge, J. A. (1969), On the subject of a flood propagation method (Muskingum method), J. Hydraul. Res., 7(2), 205-230.

Duan, Q. Y., S. Sorooshian, and R. P. Ibbitt (1988), A maximum likelihood criterion for use with data collected at unequal time intervals, Water Resour. Res., 24(7), 1163-1173.

Duan, Q. Y., S. Sorooshian, and V. Gupta (1992), Effective and efficient global optimization for conceptual rainfall-runoff models, Water Resour. Res., 28(4), 1015-1031.

Gelman, S., J. B. Carlin, H. S. Stren, and D. B. Rubin (1995), Bayesian Data Analysis, Chapman and Hall, New York.

Gurtz, J., A. Baltensweiler, and H. Lang (1999), Spatially distributed hydrotope-based modelling of evapotranspiration and runoff in mountainous basins, Hydrol. Processes, 13(17), 2751-2768.

Kavetski, D., G. Kuczera, and S. W. Franks (2006), Bayesian analysis of input uncertainty in hydrological modeling: 1 Theory, Water Resour. Res., 42, W03407, doi:10.1029/2005WR004368. 
Kennedy, M. C., and A. O'Hagan (2001), Bayesian calibration of computer models (with discussion), J. R. Stat. Soc., Ser. B, 63, 425-464.

Kloeden, P. E., and E. Platen (1992), Numerical Solution of Stochastic Differential Equations, Springer, Berlin.

Kool, J. B., and J. C. Parker (1988), Analysis of the inverse problem for transient unsaturated flow, Water Resour. Res., 24(6), 817-830.

Kuczera, G. (1983), Improved parameter inference in catchment models. 1. Evaluating parameter uncertainty, Water Resour. Res., 19(5), 1151-1162.

Kuczera, G., and E. Parent (1998), Monte Carlo assessment of parameter uncertainty in conceptual catchment models: The Metropolis algorithm, J. Hydrol., 211(1-4), 69-85.

McKay, M. D., R. J. Beckman, and W. J. Conover (1979), Comparison of 3 methods for selecting values of input variables in the analysis of output from a computer code, Technometrics, 21(2), 239-245.

Reichert, P. (2005), UNCSIM-A computer programme for statistical inference and sensitivity, identifiability, and uncertainty analysis, in Proceedings of the 2005 European Simulation and Modelling Conference (ESM 2005), edited by J. M. F. Teixeira and A. E. Carvalho-Brito, pp. 51 55, Eurosis, Ostend, Belgium.

Reichert, P. (2006), A standard interface between simulation programs and systems analysis software, Water Sci. Technol., 53(1), 267-275.

van Griensven, A., and T. Meixner (2006), Methods to quantify and identify the sources of uncertainty for river basin water quality models, Water Sci. Technol., 53(1), 51-59.

van Griensven, A., T. Meixner, S. Grunwald, T. Bishop, A. Diluzio, and R. Srinivasan (2006), A global sensitivity analysis tool for the parameters of multi-variable catchment models, J. Hydrol., 324(1-4), 10-23.

Vrugt, J. A., and W. Bouten (2002), Validity of first-order approximations to describe parameter uncertainty in soil hydrologic models, Soil Sci. Soc. Am. J., 66(6), 1740-1751.
Vrugt, J. A., H. V. Gupta, W. Bouten, and S. Sorooshian (2003), A Shuffled Complex Evolution Metropolis algorithm for optimization and uncertainty assessment of hydrologic model parameters, Water Resour. Res., 39(8), 1201, doi:10.1029/2002WR001642.

Vrugt, J. A., G. Schoups, J. W. Hopmans, C. Young, W. W. Wallender, T. Harter, and W. Bouten (2004), Inverse modeling of large-scale spatially distributed vadose zone properties using global optimization, Water Resour. Res., 40, W06503, doi:10.1029/2003WR002706.

Vrugt, J. A., C. G. H. Diks, H. V. Gupta, W. Bouten, and J. M. Verstraten (2005), Improved treatment of uncertainty in hydrologic modeling: Combining the strengths of global optimization and data assimilation, Water Resour. Res., 41, W01017, doi:10.1029/2004WR003059.

Williams, J. R. (1969), Flood routing with variable travel time or variable storage coefficients, Trans. ASAE, 12(1), 100-103.

Yang, J., K. C. Abbaspour, and P. Reichert (2005), Interfacing SWAT with systems analysis tools: A generic platform, in 2005 3rd International SWAT Conference Proceedings, edited by R. Srinivasan et al., pp. 169-178, Swiss Fed. Inst. for Aquat. Sci. and Technol., Duebendorf, Switzerland.

Yang, J., P. Reichert, K. C. Abbaspour, and H. Yang (2007), Hydrological modelling of the Chaohe Basin in China: Statistical model formulation and Bayesian inference, J. Hydrol., 340, 167-182, doi:10.1016/j.jhydrol. 2007.04.006.

K. C. Abbaspour, P. Reichert, and J. Yang, Swiss Federal Institute of Aquatic Science and Technology, Ueberlandstr. 133, P.O. Box 611, CH8600 Duebendorf, Switzerland. (jing.yang@eawag.ch) 